\title{
Tales of Health-TEA: Uma Proposta de Storytelling Digital para Conscientização do Transtorno do Espectro do Autismo
}

\author{
Ellen C. Aguiar ${ }^{1}$, Victória O. Gomes ${ }^{1}$, Victor T. Sarinho ${ }^{1}$ \\ ${ }^{1}$ Laboratório de Entretenimento Digital Aplicado - LEnDA \\ Universidade Estadual de Feira de Santana - UEFS \\ Feira de Santana - Bahia - Brasil \\ chalegreaguiar@gmail.com, victoria.oliveiragomes@gmail.com, \\ vsarinho@uefs.br
}

\begin{abstract}
This paper describes a update in the development status of a storytelling game to assist in socializing with people with Autism Spectrum Disorder (ASD). Tales of Health-TEA goal is to facilitate the inclusion of people who carries ASD in modern society, in addition to providing greater confidence in neurotypical people when dealing with those who presents behavioral peculiarities stemming from ASD.
\end{abstract}

Resumo. Este artigo descreve uma atualização sobre o estado de desenvolvimento de um jogo storytelling para auxiliar no convívio com pessoas portadoras do Transtorno do Espectro do Autismo (TEA). O objetivo do Tales of HealthTEA é facilitar a inclusão de seus portadores na sociedade moderna, além de proporcionar um maior segurança nas pessoas neurotípicas ao lidar com aquelas que apresentam peculiaridades comportamentais provindas do TEA.

\section{Introdução}

Transtorno do Espectro do Autismo (TEA) é "uma desordem neurológica que afeta o funcionamento e o desenvolvimento das áreas de interação social no comportamento e na comunicação de jovens e adultos" [Kwee et al. 2009]. Estas características compõem o tripé sintomático da perturbação autística, um espectro que tem repercussões não somente na vida das pessoas que estão nele, mas também na vida daqueles que o cercam.

"A família, como a sociedade, não foi preparada para acolher as pessoas acometidas de transtornos mentais" [Soares and Munari 2007]. Mesmo assim, "a família é inserida como parceira no processo de reabilitação psicossocial da pessoa portadora de sofrimento psíquico" [Soares and Munari 2007]. Por este motivo, diversas técnicas têm sido aplicadas na tentativa de melhorar a qualidade de vida de familiares e amigos que convivem com as peculiaridades associadas aos portadores do TEA, uma vez que saber lidar com a mesma é essencial para o bem-estar da pessoa neuroatípica. Isto é, se as pessoas ao redor do indivíduo souberem como lidar com ele, a sua qualidade de vida será melhorada significativamente.

"Storytelling é uma experiência na construção da teoria dialética em que os interlocutores constroem, criticam e reconstroem teorias de eventos mundanos" [Ochs et al. 1992], dando um significado novo a acontecimentos do dia a dia. Trata-se 
de uma abordagem que permite a análise criteriosa de decisões anteriormente tomadas, ponderando os diferentes rumos de uma história caso uma outra perspectiva fosse adotada.

Considerando a importância de se desenvolver ferramentas que se preocupem com a conscientização daqueles que convivem com indivíduos portadores do TEA [Maia Filho et al. 2016], este artigo informa sobre os avanços na confecção de um storytelling digital com contos de conscientização para os amigos, familiares e profissionais que lidam com pessoas que vivem no TEA. O mesmo já foi apresentado como proposta anteriormente [Aguiar et al. 2018], porém sofreu algumas modificações ao ser implementado, como por exemplo, a metodologia para coleta de histórias.

Tales of Health-TEA visa promover a compreensão de situações e peculiaridades comportamentais apresentados neste espectro, agrupando histórias sobre situações do dia-a-dia que envolvem portadores do transtorno, de modo a minimizar a possibilidade de que neurotípicos venham desenvolver posturas e atitudes que não contribuem para o desenvolvimento do portador do TEA ou da dinâmica familiar com o mesmo.

\section{Trabalhos Relacionados}

No contexto do storytelling digital, o mesmo já foi identificado como uma prática reflexiva que pode fortalecer comunidades [Freidus and Hlubinka 2002]. Desta forma, tem-se no storytelling uma metodologia efetiva e já introduzida com sucesso para a integração de diferentes tipos de indivíduos.

Como exemplo, uma plataforma virtual de storytelling visual para denúncias foi desenvolvida, atuando como uma forma de intervenção contra o bullying em escolas [Aylett et al. 2005]. Também foi desenvolvido um jogo touch-story para avaliar o entendimento da compreensão de narrativas em crianças com autismo usando figuras [Davis et al. 2006], o qual foi posteriormente remodelado com uso de realidade virtual para encorajar ainda mais o uso da tecnologia por este público [Ho et al. 2009].

\section{Metodologia}

Com relação ao Tales of Health-TEA, este procura apresentar várias histórias onde o jogador convive com uma pessoa que vive no TEA e escolhe como lidar com ela no contexto do cotidiano. Para que estes contos estejam corretamente apresentados e tenham caráter verídico e confiável, o mesmo será embasado em artigos, livros e teses de mestrado que apresentam estudos de caso, entrevistas e narrativas de indivíduos que vivem no TEA. Esta mudança na proposta original, que seria de coletar dados em entrevista, se deu devido a obstáculos consideráveis no desenvolvimento encontrados ao trabalhar com o público.

As histórias serão adaptadas de forma a apresentar um fluxo de interações que buscam levar a conscientizações importantes para neurotípicos. A classificação do nível de importância destas conscientizações toma como base as decisões tomadas pelo jogador no decorrer da história. Isto é, se alguém decide gritar com uma pessoa que vive no TEA, ela deve ser conscientizada para que entenda porque gritar com alguém não é o modo mais recomendado de resolver a situação. Se ela já sabe que gritar não é recomendado, não irá por este caminho e consequentemente não passará por esta conscientização.

Cada ação desempenhada pelo jogador no jogo é acompanhada de uma conscientização e, às vezes, de uma dica de qual caminho seguir. Cada conscientização 
é apresentada no jogo em forma de texto, explicando o que a pessoa fez de errado ou de certo e porque esta atitude é classificada assim. Dizer o porquê daquela ação não ser recomendada é o foco, fazendo com que o neurotípico compreenda a visão de mundo e as dificuldades que o neuroatípico possui.

Como exemplo de material de referência com histórias adaptadas para o Tales of Health-TEA, o trabalho que Lira [Lira 2004] apresenta diversas histórias que se passam em sala de aula, as quais contam sobre o processo de ensino de alguns indivíduos que vivem no TEA, sobre as interações entre alunos e professores, além das interações entre os alunos em si. Duas de suas diversas histórias já foram adaptadas para o Tales of Health-TEA, servindo como objeto de estudo para a preparação de fluxos de escolhas que conduzem a diferentes conscientizações e resultados durante uma partida.

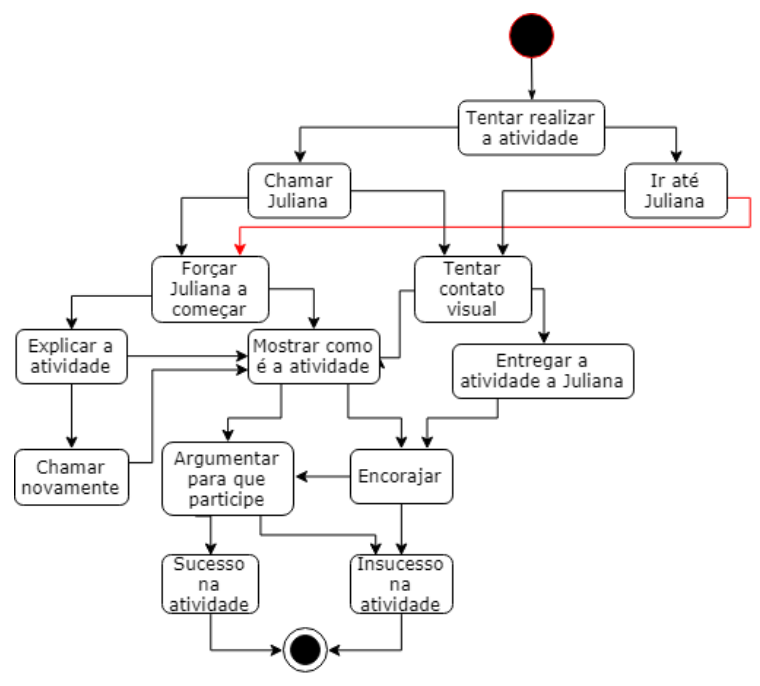

(a) Versão Telegram

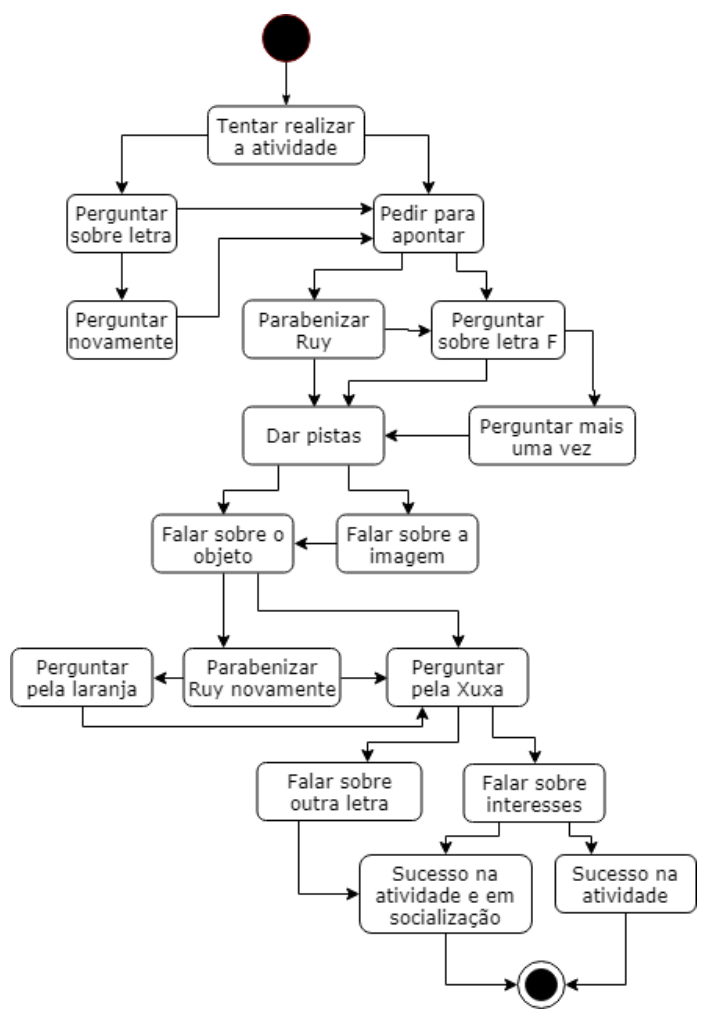

(b) Versão Desktop

Figura 1. Fluxo de decisões da primeira história adaptada.

A primeira história adaptada fala de Juliana, uma pessoa que vive no espectro autista (Figura 1(a)). Ela tem 23 anos de idade, não forma frases, apresenta gritos e usa as pessoas como ferramentas, fisicamente. A mesma também possui dificuldade em interagir e fixar o olhar, além de buscar quase sempre o isolamento. A atividade realizada é uma brincadeira com argila, visando melhorar a coordenação motora da moça. Durante a dinâmica, diversas ações são tomadas pela professora e suas interações com Juliana são estudadas, sendo justificadas ou criticadas por meio do embasamento científico sobre o TEA, suas peculiaridades comportamentais e dificuldades de aprendizagem. Cada ação vem acompanhada de uma nova informação sobre o espectro, ou algumas informações podem se repetir, quando o jogador optar por uma ação não recomendada após já ter recebido a conscientização sobre a mesma,de modo a fixar melhor este conhecimento na 
pessoa neurotípica.

A segunda história que já passou pelo processo de adaptação tem como protagonista Ruy, uma pessoa que vive no espectro autista, de 24 anos de idade que apresenta fala ecolálica (Figura 1(b)). O mesmo também demonstra risos imotivados, tende ao isolamento, possui dificuldade em fixar o olhar e apresenta comportamento estereotipado. A atividade realizada é a associação de letras com objetos que começam com as mesmas e também com o som desta letra. Para isto, Ruy é incentivado a interagir com um mural que possui letras em cima e figuras de objetos de seu cotidiano embaixo. Letras e figuras são organizados por associação, ou seja, o objeto presente na figura de baixo inicia com a letra que está acima dele. A professora tenta fazer com que Ruy lhe mostre diferentes letras e figuras, associando-as aos poucos. Novamente, esta dinâmica se torna objeto de estudo e cada interação entre aluno e professora é estudada, podendo ser justificada ou criticada.

Nesta história, também temos a participação do colega de Ruy, Danilo. Danilo é um garoto de 12 anos de idade que vive no espectro autista. O mesmo também não se comunica, embora possa, pois diz algumas palavras quando sente vontade. Assim como os demais, retrata dificuldade de fixar o olhar e interagir com as pessoas, porém possui o diferencial de emitir sorriso social. Danilo também apresenta comportamento estereotipado, mais especificamente, bate com objetos em outros objetos. As interações entre Danilo e Ruy são poucas, porém estudadas tão a fundo quanto as interações dos alunos com a professora. O jogo considera importante mostrar que as interações entre neuroatípicos também são delicadas e possuem suas razões e peculiaridades.

Com relação a execução das histórias de conscientização modeladas, a ideia é que cada história configurada seja dividida em cards que: 1) relatem algo que esteja ocorrendo de modo a contextualizar o jogador na história contada; 2) forneçam opções de tomada de decisão para o jogador; e 3) processem a decisão tomada pelo jogador de modo a interpretar novos cards, gerando como resultado novos contextos para o jogador e mantendo como consequência o fluxo de execução do jogo.

Como este ciclo de execução apresentado se encaixa com o game loop de jogos estilo quiz, utilizou-se a plataforma AsKME [Sarinho et al. 2018] para fins de processamento dos cards configurados para o Tales of Health-TEA. AsKME é capaz de executar configurações de jogos estilo quiz em múltiplas plataformas alvo, tais como desktop, web, chatbots de Mensagens Instantâneas (IM) [Lebeuf et al. 2018] e Arduino, bem como ser adaptado para executar outros gêneros de jogos, desde que sigam o ciclo de execução de mostrar perguntas/histórias e processar respostas dos jogadores.

Como resultado, um protótipo do Tales of Health-TEA foi implementado para múltiplas plataformas de execução (Figura 2), fazendo com que o jogador tenha acesso as histórias configuradas nos cards em diferentes ambientes, dentro de uma interface simples e representativa do jogo.

\section{Conclusões e Trabalhos Futuros}

Este artigo apresentou a proposta de um jogo storytelling para a conscientização de pessoas neurotípicas das peculiaridades de uma pessoa que vive no TEA. Ao fornecer aos neurotípicos um maior entendimento sobre o comportamento de portadoras do TEA, 


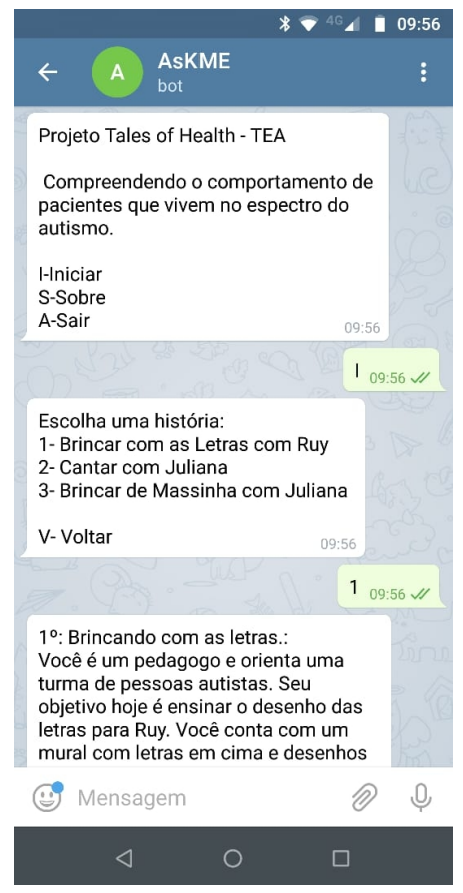

(a) Versão Telegram

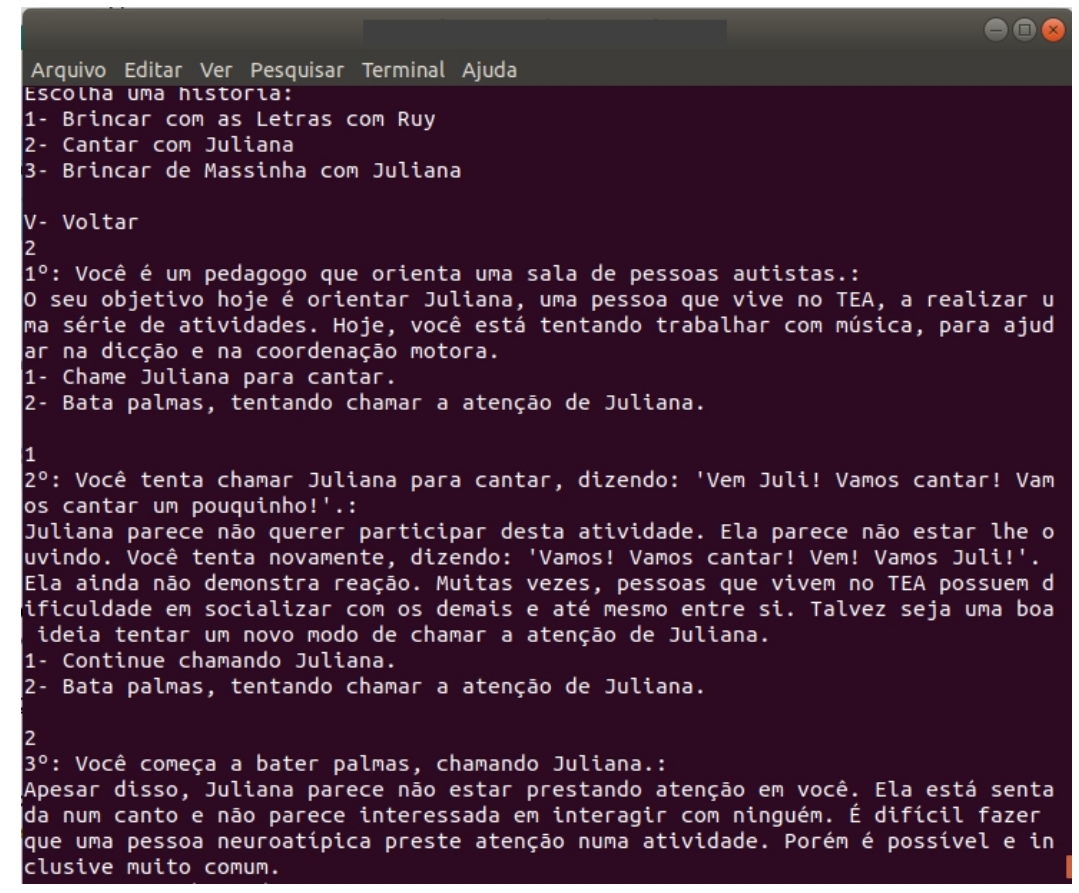

(b) Versão Desktop

Figura 2. Telas do Tales of Health-TEA executado na plataforma AsKME.

almeja-se conscientizar e direcionar a conduta dos mesmos, de modo a contribuir significativamente para uma melhoria na qualidade de vida dos que convivem com o TEA.

Com relação a importância do jogo, este permite a conscientização aberta e gratuita para todos com acesso a recursos tecnológicos, tais como web ou IM, aumentando assim o alcance e facilitando o entendimento da proposta do jogo. Mais ainda, a construção de cada história vivenciada pelo jogador é feita pelo jogo e pelo jogador, conforme o mesmo escolhe a rota da história pela qual deseja seguir. Como resultado, quem menos entende do TEA, ou mais especificamente, quem escolher as ações consideradas "não recomendadas" de uma maneira mais frequente, irá passar por mais "etapas" de conscientização, e consequentemente por mais conhecimento sobre como lidar com o TEA no cotidiano.

Contudo, é válido salientar que indivíduos que vivem no TEA possuem peculiaridades e personalidades únicas. Ou seja, apesar das situações e reações contadas no jogo serem teoricamente embasadas para a maioria destes indivíduos, não existe uma garantia de que todos apresentarão a mesma reação a tais estímulos. Assim sendo, o jogo carrega sua própria porcentagem de erro baseada no fator humano que o cerca.

Como trabalhos futuros, após a finalização da primeira versão do jogo, mais histórias serão acrescentadas, a exemplo do "como lidar com uma pessoa que vive no TEA em seu ambiente de trabalho". Elas irão descrever situações mais específicas, ampliando o alcance da conscientização para outras faixas etárias e situações vivenciadas. Também é importante realizar em um futuro próximo testes de avaliação da usabilidade do jogo produzido, o tempo gasto em cada história e se o mesmo é aceitável. Não obstante, é preciso melhorar o engajamento do público. Para isto, pretende-se a produção de 
recursos multimídia e a divulgação dos resultados em mídia social.

\section{Agradecimentos}

Agradecemos ao Programa de Bolsa de Iniciação Científica da Universidade Estatual de Feira de Santana (PROBIC/UEFS) pela concessão da Bolsa de Pesquisa para este projeto. A mesma se mostrou fundamental na jornada de desenvolvimento. Também agradecemos ao apoio e auxílio da professora Flávia Andrade Brito de Castro, pedagoga do CEB/UEFS (Centro de Educação Básica da Universidade Estadual de Feira de Santana), que se dispôs a oferecer sua orientação e conselhos para este projeto.

\section{Referências}

Aguiar, E. C., Gomes, V. O., and Sarinho, V. T. (2018). Projeto tales of health: Uma proposta de jogo digital para conscientização do transtorno do espectro do autismo. XVII Brazilian Symposium on Computer Games and Digital Entertainment (SBGames).

Aylett, R. S., Louchart, S., Dias, J., Paiva, A., and Vala, M. (2005). Fearnot!-an experiment in emergent narrative. In International Workshop on Intelligent Virtual Agents, pages 305-316. Springer.

Davis, M., Dautenhahn, K., Nehaniv, C., and Powell, S. (2006). Towards an interactive system eliciting narrative comprehension in children with autism: A longitudinal study. In Designing accessible technology, pages 101-114. Springer.

Freidus, N. and Hlubinka, M. (2002). Digital storytelling for reflective practice in communities of learners. ACM SIGGROUP Bulletin, 23(2):24-26.

Ho, W. C., Davis, M., and Dautenhahn, K. (2009). Supporting narrative understanding of children with autism: A story interface with autonomous autobiographic agents. In Rehabilitation Robotics, 2009. ICORR 2009. IEEE International Conference on, pages 905-911. IEEE.

Kwee, C. S., Sampaio, T. M. M., and Atherino, C. A.-a. C. A. A. T. (2009). Autismo: uma avaliação transdisciplinar baseada no programa TEACCH. Revista CEFAC.

Lebeuf, C., Storey, M.-A., and Zagalsky, A. (2018). Software bots. IEEE Software, $35(1): 18-23$.

Lira, S. M. d. (2004). Escolarização de alunos autistas: Histórias de sala de aula. Master's thesis, Universidade do Estado do Rio de Janeiro.

Maia Filho, A. L. M., Amanda, L. A. N. M. N., Nogueira, L., Silva, K. C. O., and Santiago, R. F. (2016). A importância da família no cuidado da criança autista/the importance of the family in the care of autist children. Saúde em Foco, 3(1):66-83.

Ochs, E., Taylor, C., Rudolph, D., and Smith, R. (1992). Storytelling as a theory-building activity. Discourse processes, 15(1):37-72.

Sarinho, V. T., de Azevedo, G. S., Boaventura, F. M., and de Santana, F. (2018). Askme: A feature-based approach to develop multiplatform quiz games. In XVII Brazilian Symposium on Computer Games and Digital Entertainment (SBGames).

Soares, C. B. and Munari, D. B. (2007). Considerações acerca da sobrecarga em familiares de pessoas com transtornos mentais. Ciência, Cuidado e Saúde, 6(3):357-362. 